\title{
Recomendaciones prácticas para evitar el desacondicionamiento físico durante el confinamiento por pandemia asociada a COVID-19
}

\author{
Practical recommendations to avoid physical deconditioning during confinement due to pandemic associated with \\ COVID-19
}

\author{
Andry Yasmid Mera-Mamián ${ }^{1,2 *}$ orcid.org/0000-0002-2356-3370 \\ Esteban Tabares-Gonzalez' orcid.org/0000-0003-2474-0292 \\ Santiago Montoya-Gonzalez' orcid.org/0000-0001-6608-6458 \\ Diana Isabel Muñoz-Rodriguez 1 orcid.org/0000-0003-4255-4813 \\ Felipe Monsalve-Vélez' orcid.org/0000-0002-6852-8843
}

1 Facultad de Fisioterapia, Universidad CES. Medellín, Colombia

2 Centro afiliado Cochrane, Universidad del Cauca. Popayán, Colombia

\section{Resumen}

Introducción: El aislamiento social durante la pandemia por COVID-19 incluye el confinamiento en casa, que redunda en incremento de la inactividad física y de comportamientos sedentarios, favoreciendo el desacondicionamiento físico. Las personas desacondicionadas físicamente tienen alteraciones metabólicas y sistémicas por la falta de movimiento. Objetivo: Presentar recomendaciones prácticas, y de bajo costo sobre actividades basadas en la evidencia para evitar el desacondicionamiento físico durante el confinamiento en casa, que se pueden mantener a largo plazo, incluso después de la pandemia. Materiales y métodos: Revisión documental sobre estrategias para conducir estilos de vida saludable en casa que disminuyan los efectos negativos a nivel fisiológico producidos por el confinamiento derivado de la pandemia. Resultados: La evidencia señala que el confinamiento en casa incrementa los niveles de inactividad física y el comportamiento sedentario; la realización de actividad física bajo condiciones particulares puede entre otros, fortalecer el sistema respiratorio e inmunológico, mantener la condición física y generar efectos positivos sobre la salud mental. Conclusiones: Los hábitos relacionados con la práctica de actividad física en casa se deben mantener en el tiempo, se recomienda realizarla de manera programada, establecer horarios de descanso entre las actividades y durante la noche, y elegir aquellas que generen disfrute.

Palabras clave: Actividad física; conductas sedentarias; pandemia; aislamiento social; coronavirus. (Fuente: DeCS, Bireme).

\begin{abstract}
Introduction: Social isolation during the COVID-19 pandemic includes home confinement, which results in increased physical inactivity and sedentary behaviors, favoring physical deconditioning. Deconditioned people have metabolic and systemic disorders due to the lack of movement. Objective: To present practical, low-cost recommendations on evidence-based activities to avoid physical deconditioning during home confinement, which can be maintained over the long term, even after the pandemic. Materials and methods: Documentary review on strategies to support healthy lifestyles at home that decrease the negative effects at a physiological level produced by the confinement derived from the pandemic. Results: Evidence indicates that home confinement increases the levels of physical inactivity and sedentary behavior; executing physical activity under particular conditions, among other effects, can strengthen the respiratory and immune systems, maintain physical condition and generate positive effects on mental health. Conclusions: The habits related to the practice of physical activity at home should be maintained over time, it is recommended to do it on a scheduled basis, define rest times between activities and during the night, and choosing those that generate enjoyment.
\end{abstract}

Key words: Physical activity; sedentary behaviors; pandemic; social isolation; coronavirus. (Source: DeCS, Bireme).

*Autor de correspondencia

Andry Yasmid Mera-Mamián

e-mail: mera.andry@uces.edu.co 


\section{Introducción}

La pandemia por coronavirus COVID-19 declarada el 11 de marzo de 2020 por la Organización Mundial de la Salud (OMS)(1), ha llevado a que varios países adopten medidas no farmacológicas y combinadas(2), para contener y mitigar el contagio en sus poblaciones, siendo una de las medidas principales el aislamiento social(3); en este, se incluye el confinamiento en casa con el objetivo de favorecer el distanciamiento, dado que el contacto cercano con personas infectadas, aumenta la probabilidad de contagio(4). Estas medidas no son inocuas y la restricción de la participación en actividades al aire libre, así como los desplazamientos a escuelas, universidades y sitios de trabajo, reducen el tiempo dedicado a la realización de Actividad física (AF) y

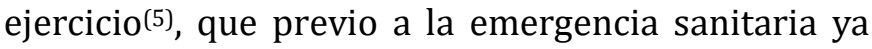
era considerado también como un grave problema de salud pública mundial(6), favoreciendo la aparición de efectos deletéreos asociados a la inactividad física, el sedentarismo, el incremento en el consumo de alimentos con alto aporte calórico, bebidas alcohólicas, la mala calidad del sueño(5,7), condiciones que en conjunto favorecen el desacondicionamiento físico.

Este desacondicionamiento es un síndrome que aparece en personas con reposo prolongado (inactividad física y conductas sedentarias), su principal característica es la atrofia muscular que afecta principalmente las fibras musculares tipo II y se asocia a un daño de la placa neuromuscular. Esto conlleva a múltiples alteraciones metabólicas y sistémicas derivadas de la falta de movimiento ${ }^{(8,9)}$.

Por tanto, abordar estrategias para eliminar la probabilidad de desacondicionarse físicamente es vital, puesto que cuando se está activo los músculos producen compuestos que mejoran el funcionamiento del sistema inmunitario, fisiológico y biológico en general. Además, ya se ha documentado ampliamente que la AF es esencial para prevenir y tratar enfermedades crónicas, así como para controlar las condiciones de estrés a las que está enfrentada la población mundial por el impacto devastador de esta emergencia en términos sanitarios, sociales, culturales y económicos(10). Estas condiciones de estrés conducen a su vez a mala calidad del sueño y alteraciones relacionadas con la salud mental(11).
Hasta ahora, la población conoce de los efectos benéficos de la AF y de los dominios en los que se puede trabajar en esta: tareas domésticas, actividades recreativas, las relacionadas con el trabajo y como medio de transporte(12); sin embargo, estos tres últimos dominios estarán prohibidos mientras permanezca la estrategia de aislamiento social para controlar el contagio por coronavirus $(2,13)$. Por tanto, se hace necesario develar potenciales estrategias de AF y hábitos saludables que puedan ser adoptadas desde el confinamiento en casa por el núcleo familiar.

Adoptar de manera temprana estrategias que eviten las complicaciones propias de la pandemia derivadas del aislamiento social, y basados en recomendaciones sobre intervenciones efectivas, sencillas, de bajo costo y sostenibles en el tiempo, pueden favorecer que muchas de estas se conviertan en hábitos y que su práctica perdure a largo plazo, contribuyendo de esta manera al control tanto de la probabilidad de contagio por COVID-19(10), como a la aparición de condiciones crónicas(12).

Considerando que el abordaje del desacondicionamiento físico incluye otros elementos además de la $\mathrm{AF}$, esta revisión documental incluye los estilos de vida saludable relacionados con nutrición, sueño y salud mental, así como las recomendaciones de AF, alimentación y sueño. Este documento puede servir de base de consulta para investigadores, tomadores de decisiones y población general. Estas recomendaciones, no consideran población con infección por COVID-19 sintomáticas, dado que estas personas requieren indicaciones particulares, según severidad y sintomatología asociada.

Para aportar en la reducción de estas consecuencias, se planteó el desarrollo de una revisión de literatura que permitiera consolidar algunas recomendaciones de expertos referentes en el área de la salud. Por tanto, el objetivo principal de esta revisión es presentar recomendaciones prácticas y de bajo costo sobre actividades basadas en la evidencia para evitar el desacondicionamiento físico, durante el confinamiento en casa, actividades que se pueden realizar de manera individual o en familia $e$ involucrar en las rutinas diarias, ya sea de manera parcial o permanente y mantenerse a largo plazo, incluso después de la pandemia. 


\section{Materiales y métodos}

Se realizó búsqueda de literatura utilizando las palabras clave en español y en inglés: actividad física, conducta sedentaria, pandemia, aislamiento social, coronavirus, acondicionamiento físico., confinamiento y COVID-19. Se realizó búsqueda en Medline a través de Pubmed y Google académico, se incluyeron páginas oficiales de la Organización Mundial de la Salud (OMS), la Organización Panamericana de Salud (OPS), el Ministerio de Salud y Protección Social colombiano y referentes en el área de la $\mathrm{AF}$ de nivel nacional e internacional. También se consideró recomendaciones del equipo de investigadores que incluye expertos en el área de la AF y deporte. Se incluyó documentos en español e inglés. Se excluyó documentos que presentaban recomendaciones desactualizadas a la luz de la evidencia o ambiguas, además de aquellas que implican actividades fuera de casa y para pacientes positivos para COVID-19.

\section{Resultados}

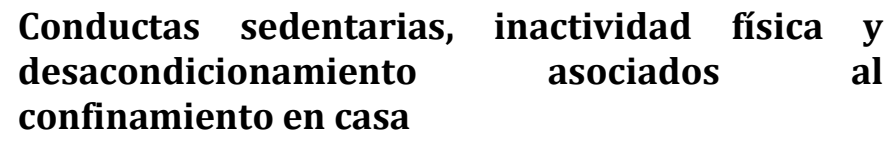

Bajo la indicación de permanecer en casa se incrementa de manera importante el tiempo que se permanece sentado o recostado, realizando actividades académicas, laborales (teletrabajo) y de ocio, que requieren poco gasto de energía, lo cual se conoce como sedentarismo o comportamiento sedentario(14,15). A largo plazo, esto se ha asociado con desacondicionamiento físico (síndrome que combina cambios como pérdida de masa muscular, disminución de la flexibilidad y de la fuerza) y la aparición de enfermedades crónicas no transmisibles (ECNT) como obesidad, diabetes y enfermedades cardiovasculares, otra de las pandemias de la actualidad(16), incrementando la morbilidad general. La OMS describe que "el comportamiento sedentario y los bajos niveles de $A F$ pueden tener efectos negativos en la salud, el bienestar y la calidad de vida de las personas"(17).

\section{Disminución del gasto calórico durante el confinamiento en casa}

El exceso de calorías ya sea por incremento en el consumo de harinas, grasas y azúcares o por el bajo gasto de estas por disminución en los niveles de AF, puede favorecer el incremento rápido y no deseado del peso corporal, y consecuentemente la aparición de complicaciones relacionadas con el estado nutricional $(18,19)$ Además, la mala alimentación, se ha descrito como un factor que puede deteriorar la salud bucal y esta a su vez afectar negativamente la calidad de vida de las personas(20).

Asimismo, la OMS ha reportado la obesidad en niños y adolescentes como un problema de salud pública. Para 2017 el número de personas obesas entre 5 y 19 años a nivel mundial, se incrementó casi 10 veces, respecto a los cuatro últimos decenios(21). En Colombia según reporte del Ministerio de Salud y Protección Social del año 2019, la prevalencia de exceso de peso en menores de 18 años fue de $17,53 \%$ (2.7 millones de personas)(22). Es muy probable que estas cifras sigan en aumento durante la estancia prolongada en casa, si no se adoptan medidas adecuadas para prevenirlo.

\section{Mala calidad del sueño y alteraciones relacionadas con la salud mental, durante el confinamiento en casa}

La falta de un descanso adecuado durante la noche, asociado a diferentes situaciones estresantes durante el periodo de confinamiento, puede favorecer la aparición de fatiga durante el día(7) y la agudización de problemas como estrés, ansiedad y depresión(23).

El aislamiento social, en general, conlleva a disminución en el contacto con otras personas y cambios de hábitos relacionados con actividades familiares, académicas, laborales y sociales, pudiendo presentarse episodios de ansiedad, que pueden agravarse por la información que se recibe constantemente respecto a condiciones externas(24). Un estudio realizado en China en las fases iniciales de la pandemia, reportó que en más del $95 \%$ de los participantes, fue la internet la principal fuente de información(25), dato relevante si se tiene en cuenta que un alto porcentaje de la información que se carga en la web puede ser confusa o falsa ${ }^{(26)}$.

\section{Beneficios de la actividad física en el sistema respiratorio y el sistema inmune}

Algunos estudios han reportado que la AF moderada (3-6 METs, por sus siglas en inglés Metabolic equivalent of task) como caminar a paso rápido, bailar y hacer actividades domésticas, tienen un impacto positivo en la función del sistema inmune, efecto que se reduce parcialmente cuando se realizan ejercicios vigorosos ( $>6$ METs) como desplazamiento de cargas pesadas (>20 kg), 
ciclismo o maratones(27-29). Este podría no ser variable en el contexto del hogar, dado que el menor efecto de la AF vigorosa, se ha descrito solo en contextos de competencia (poco descanso, mala alimentación, estrés, aglomeración de personas y alta movilidad entre ciudades o países) ${ }^{(20,30,31) .}$

Dentro de los beneficios descritos tanto en el sistema inmune como en respiratorio, se encuentran la reducción de la severidad de enfermedades infecciosas( ${ }^{(32)}$, principalmente, a través de la disminución de procesos inflamatorios(27,33), mejora en las condiciones de personas con ECNT como diabetes, alteraciones cardiacas y cáncer, atenuando el impacto negativo que las enfermedades infecciosas podrían tener sobre estas poblaciones(10,33); disminución del riesgo de infecciones respiratorias del tracto superior(28) y regulación de los niveles de cortisol, con lo cual se favorece la disminución de los niveles de estrés, control de la ansiedad y mejora en el funcionamiento del sistema inmune y del metabolismo(34,35). La AF se convierte en un aliado para el fortalecimiento del sistema inmune y respiratorio en la población general(5).

\section{Recomendaciones prácticas que se pueden implementar durante el confinamiento en casa para evitar el desacondicionamiento físico y conservar en el tiempo}

En el contexto de la pandemia, las recomendaciones que previamente ha generado la $\mathrm{OMS}^{(29)}$ y la guía de actividad física del Departamento de Salud y Servicios Humanos de los Estados Unidos(36), deberán ser ajustadas, principalmente a actividades que se pueden realizar dentro del hogar, ya sea de manera individual o con el grupo familiar. En este sentido, las actividades domésticas que hacen parte de la AF cotidiana como limpiar, lavar, cocinar, deben mantenerse (AF no formal)(37).

La primera recomendación general es definir y delimitar objetivos claros, según el nivel de entrenamiento previo para facilitar la elección de las actividades a incorporar. Establecer un cronograma de actividades diarias o semanales, en las que se incluya las diferentes actividades académicas, laborales, domésticas, de AF, relajación y ocio; el límite de tiempo entre cada una de ellas, será importante para favorecer su cumplimiento y evitar la fatiga.

1. Respecto a actividades académicas y laborales a) Quienes desarrollen actividades académicas o laborales en casa, disponer un espacio tranquilo, limpio y ordenado, realizando desinfección frecuentemente( ${ }^{(38)}$. Esto favorece la preparación y disposición para el desarrollo de las diferentes actividades, evitando tensiones innecesarias.

b) En el caso de las personas que desarrollan sus actividades a través del uso de un computador, mantener la espalda recta, con sus codos y cadera en flexión de $90^{\circ}$ y con la pantalla del computador a una altura tal, que la parte superior del equipo proyecte justo al frente de los ojos (para lograrlo se podrá hacer uso de bancos, libros o cualquier elemento estable disponible en casa)(39). Su utilidad se basa en la disminución de dolores en espalda y extremidades.

c) Tomar espacios de pausas activas entre las diferentes actividades (cada 30 minutos, máximo cada hora) con una duración entre 3 y 5 minutos, para favorecer la movilización de las extremidades y la circulación sanguínea. Ponerse de pie y realizar un desplazamiento corto, involucrando algún tipo de estiramiento(17). Se recomienda hacer uso de una alarma que le recuerde los tiempos para tomar la pausa.

2. Incremento de los niveles de actividad física y disminución del sedentarismo

a) Incluir la realización de AF, mínimo 60 minutos diarios para niños y adolescentes entre 5 y 17 años y 150 minutos semanales para mayores de 18 años. Recordando que un incremento en este tiempo, se traduce en mejores resultados para la salud(29).

b) Aprovechar los programas de entrenamiento en línea para seguir una rutina e incorporar diferentes actividades. Es importante considerar su nivel de entrenamiento previo y empezar con rutinas sencillas, luego incrementar la complejidad o el tiempo progresivamente, de esta manera se evitan lesiones(17).

c) Si se ha planeado una rutina de ejercicios específicos, previamente se deberá realizar adecuado calentamiento con movilización articular, activación y estiramientos dinámicos controlados (lentos) de la musculatura, esto mejora el rendimiento y podría evitar lesiones, volviendo más 
eficiente la contracción muscular (más energía con menos fatiga)(12).

d) Se recomienda incorporar ejercicios para mejorar la respuesta muscular como sentadillas, flexiones de pecho o abdominales, que involucran grandes grupos musculares. Este tipo de actividades se deberán realizar mínimo 2 veces por

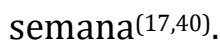

e) Participar en las actividades del hogar, hacer aseo, cocinar, organizar, también cuenta como AF. Involucrar a los integrantes del hogar facilita la distribución de cargas y libera espacios de esparcimiento para todos $(29,37,40)$.

f) Es posible utilizar elementos del hogar como pesas, durante las diferentes actividades, es importante asegurarse que sean objetos seguros; que se puedan agarrar bien, que no tengas filos que puedan cortar, o texturas que puedan lastimar la piel y que tengan un peso acorde a su edad, estado de salud y condición física previa (37).

g) Para favorecer que las caminatas dentro de la casa se tornen dinámicas y permitan la activación de diferentes grupos musculares, se recomienda aprovechar las escaleras o crear circuitos dentro de la casa, con estaciones, variando por ejemplo entre caminata, trote y salto $(17,38)$.

h) Hidratarse constantemente, según los niveles de intensidad de la actividad que realice, preferiblemente con agua, evitando el consumo frecuente de bebidas azucaradas y/o alimentos procesados(41).

i) Posterior a la realización de la rutina de ejercicios, realizar estiramientos musculares de 30 segundos sostenidos cada uno(12), esto evitará que se presente fatiga muscular y lesiones.

j) Durante la realización de $\mathrm{AF}$, es muy importante que se incorpore una respiración adecuada y profunda, procurando que al tomar el aire, se expanda el abdomen y no el pecho (respiración diafragmática)(37).

k) Para las personas mayores de 65 años (adultos mayores) con movilidad reducida, se sugiere incluir actividades de equilibrio y propiocepción tres o más días a la semana, a fin de prevenir caídas; es muy importante la vigilancia y acompañamiento constante(29).
También se deben incluir sesiones de estiramiento diarias.

l) Si el ejercicio genera molestias o no se tolera, se deberá suspender. Asimismo, tener especial cuidado y realizar actividad de baja intensidad en personas con alteraciones músculo esqueléticas recientes, afecciones cardiopulmonares o patologías orgánicas graves $^{(42) .}$

m) Es muy importante que la AF que se elija para mantener las condiciones de salud, sea una actividad con la que se sienta a gusto y se disfrute en compañía de la familia, o con las personas con quienes esté asumiendo el aislamiento preventivo, con el fin de que se continúe realizando cuando finalice la pandemia. Si las personas son sedentarias lo más importante es involucrarse con la AF, el tiempo y la intensidad se irán incrementando gradualmente hasta obtener los niveles recomendados, "cualquier movimiento cuenta para la buena salud" (43,44).

\section{A nivel nutricional}

a) Disminuir el consumo de harinas y grasas e incrementar el consumo de frutas y verduras, dado que la demanda calórica disminuye con la reducción de los desplazamientos, la inactividad física y el sedentarismo.

b) Evitar el consumo de alimentos procesados y comidas rápidas, debido a su elevada densidad energética(45). Incrementar el consumo de granos enteros, puesto que los cereales y legumbres son la base de la alimentación. Es importante incluir en la dieta pan, pasta y arroz, (preferiblemente integrales), $y$ legumbres guisadas $o$ estofadas, tratando de cocinar estos alimentos con verduras(46).

c) Disminuir el consumo de sal y azúcar(47-49), ya que no está recomendado en general, y por lo tanto, tampoco en períodos de aislamiento social debido a que pueden aumentar el riesgo de padecer sobrepeso $u$ obesidad(45) y otras patologías asociadas. La disminución de ejercicio físico y el incremento de las conductas sedentarias y una dieta no balanceada podría aumentar el riesgo de padecer enfermedades crónicas(50).

d) Preferir alimentos cocidos y preparados al vapor, disminuyendo el consumo de aquellos horneados o fritos, ya que la cocción al vapor 
favorece la conservación de minerales y vitaminas(47,51).

e) Se recomienda tener hábitos alimenticios saludables, evitando dietas estrictas sin prescripción de un profesional, dado que esto puede representar riesgo para la salud(49).

f) Es necesario garantizar el consumo de agua a demanda (según la sensación de sed) o incluso sin dicha sensación (en personas jóvenes y mayores), al menos 1,8 litros de líquido al día, prefiriendo siempre el agua como fuente de hidratación. De forma complementaria, incluir caldos o sopas, infusiones, té y favorecer una ingesta de frutas y verduras que también contribuyen al aporte de agua(52).

4. Relacionadas con la salud mental

a) Incluir programas de meditación y relajación online que puedan aportar actividades o ideas para realizar en casa(53). Es importante adecuar un espacio tranquilo $\mathrm{y}$ bien ventilado, de tal manera que se eviten interrupciones y se culminen las actividades con éxito. Lo anterior, debido a que la meditación ha mostrado efectos benéficos en el tratamiento del dolor crónico, la ansiedad, ataques de pánico, estrés, insomnio, trastornos de la conducta alimentaria; alteraciones que podrán estar relacionadas con el aislamiento social prolongado(54).

b) Juegos de memoria o concentración. La realización de este tipo de actividades, involucrando a la familia (en la medida de lo posible), ya sea de manera virtual o presencial, favorecen la activación de las funciones cognitivas y la liberación de tensión. Es necesario que se procure mantener las rutinas $\mathrm{y}$ los horarios habituales, en particular las actividades de aprendizaje, así como momentos para jugar de forma segura y relajarse(55).

c) Dentro de las posibilidades particulares se debe incluir dentro de la programación, tiempos adecuados de descanso en la noche (entre 7 y 8 horas), preferiblemente manteniendo distantes equipos electrónicos(45). Lo anterior debido a que el sueño es una función biológica fundamental determinante del estado de salud físico y psicológico de la persona, interviniendo en la restauración neurológica(56); facilitar el restablecimiento de la energía celular, así como los procesos inmunológicos y preservar la memoria(57). Además, a nivel psicológico es esencial para el bienestar mental y la calidad de vida(58).

d) Si se requiere consultar información sobre COVID-19 y las medidas adoptadas en la región, se sugiere acceder a fuentes oficiales para evitar información falsa que pueda incrementar los niveles de angustia(9), estableciendo horarios determinados para esto, evitando saturarse con la gran cantidad de datos que se publica diariamente.

e) Mantenerse conectado con familiares y amigos, planeando actividades online para distraerse y dar o recibir apoyo emocional durante el aislamiento(53).

f) Reconocer las señales de estrés como: dificultad para dormir, aumento del consumo de alcohol y otras sustancias ilícitas, mal genio frecuente, sensación de depresión, poca energía(53). En estos casos será importante implementar estrategias oportunas de control o contactar ayuda psicológica, en caso de que los síntomas sean más complejos.

\section{Discusión}

La pandemia por COVID-19 ha modificado rápidamente los modos de vida de la población en general, y la ausencia de vacunas y tratamientos específicos para COVID-19 exhortan la necesidad de emplear estrategias públicas dirigidas a reducir las tasas de contactos en la población y reducir la transmisión del virus(2). Dentro de la fase de mitigación en la que se encuentra el país (Colombia), el confinamiento en casa se ha adoptado como medida obligatoria, y aunque su principal objetivo es reducir el contagio, implica otros riesgos para la salud, tales como los mentales (estrés, ansiedad, alteraciones de sueño)(59), y físicos; incluido en este segundo grupo, el desacondicionamiento físico, dado entre otros, por el incremento de los tiempos de inactividad física, las conductas sedentarias y la malnutrición $(8,9)$.

Sin embargo, es posible evitar esta condición durante el confinamiento y mejorar los hábitos; incluso, adquirirlos si no se tenían previamente, pues algunos elementos como la compañía de la familia 
pueden jugar un papel importante en mantenerlos, aun cuando termine la pandemia, constituyéndose en hábitos saludables(60,61). Esta revisión, presentó un listado de recomendaciones que se considera, pueden de manera conjunta, disminuir los efectos negativos asociados al desacondicionamiento físico por falta de movilidad y de algunos cuidados generales.

Ante la necesidad de contener la propagación del virus y mitigar la rápida desaceleración de los procesos organizacionales, aparece el teletrabajo (trabajo remoto), una de las actividades más frecuentes durante la pandemia(62,63), que promueve mayor tiempo frente a pantallas y menos tiempo para hacer AF. Además, se ha señalado que este puede incrementar los niveles de estrés y ansiedad de manera importante, especialmente por la rapidez con que se debió implementar la medida, y porque en muchos casos, no se tienen todos los conocimientos necesarios en el uso de herramientas virtuales; no estar preparado para trabajar en casa implica que posiblemente no se logren establecer horarios específicos de inicio y culminación de actividades laborales, con potenciales riesgos psicosociales para los trabajadores(63), que, combinado con la incertidumbre de la situación sanitaria, económica, social y cultural, redunda en menor productividad e incremento en las condiciones de morbilidad de la población, tanto físicas como mentales(24,64). Por eso, las recomendaciones descritas apuntan a que la población no incurra en tensiones innecesarias adicionales.

La AF realizada de manera libre ya sea sin conocimiento previo o sin algún tipo de asesoramiento, puede favorecer la aparición de lesiones en diferentes tejidos(65), conllevando a menor adherencia. Por tanto, cuando se planea la realización de $\mathrm{AF}$, considerar recomendaciones tales como el calentamiento y los estiramientos musculares de manera adecuada, favorecen una mejor condición de salud(12). Además como se menciona en las recomendaciones, la prescripción del ejercicio en personas con comorbilidades deben considerarse para evitar lesiones; en este caso, los ejercicios deberán realizarse bajo indicación de un profesional de la salud capacitado, lo que le permita integrar adecuadamente los beneficios científicamente comprobados de la $\mathrm{AF}^{(66)}$.
Si bien la evidencia empírica hasta ahora es escasa sobre los efectos de la AF en la disminución del riesgo de infección por COVID-19, hay suficiente evidencia que demuestra que el ejercicio físico, definido como "AF planificada, estructurada, repetitiva y realizada con un objetivo relacionado con la mejora o el mantenimiento de uno o más componentes de la aptitud física"(29), protege a las personas ante diferentes infecciones de tipo viral; se ha documentado que el ejercicio puede mitigar los efectos negativos del estrés y mantener la función inmune, particularmente en personas sometidas a periodos largos de aislamiento y confinamiento como los presentados por la pandemia actual(67).

Es clara la importancia de mantener niveles adecuados de AF dentro de las pautas recomendadas, no solamente por el efecto positivo en las células y moléculas del sistema inmune, sino que este realizado de manera regular, genera efectos tanto físicos como mentales, a la vez que se presenta como una alternativa para disminuir el tiempo en pantalla(68), que se puede ver incrementado al permanecer más tiempo en casa, y que en exceso impacta negativamente las habilidades cognitivas y favorece desenlaces no deseados como la obesidad y la depresión(69). Las actividades prácticas para disminuir el tiempo en sedente pueden mejorar incluso el estado de ánimo( ${ }^{(70)}$ y favorecer la toma de decisiones de manera más clara y organizada.

Se ha señalado que se debe prestar especial atención en salud a aquellas personas que dejan de trabajar durante el aislamiento $y$ aquellas que eran físicamente activas $y$ debieron suspender $o$ disminuir su nivel de AF, puesto que podrían ver más afectada su condición de bienestar general(11). En el caso de los adultos mayores, uno de los principales objetivos será disminuir el riesgo de caídas, dado que esto puede implicar mayor demanda de los servicios de salud(71), y limita el cumplimiento del aislamiento establecido particularmente para esta población, dado que son considerados más vulnerables(72).

Los problemas de salud mental ante una pandemia suelen ser agudos; sin embargo, es posible observar secuelas emocionales a largo plazo(73), ya que está el constante temor por el contagio sumado a la incertidumbre y las sensaciones negativas como el aburrimiento, por lo que es necesario, la incorporación de actividades constantes que permitan mantener una buena condición mental. La 
salud mental en condiciones adversas como las desencadenas a la luz de la pandemia, permiten entre otros, que la mejora los procesos fisiológicos y que el cuerpo se disponga de manera adecuada para las diferentes actividades que se programan en el día a día(74). Es necesario prestar especial atención a grupos emocionalmente vulnerables (personas con enfermedades de salud mental), en quienes se ha encontrado relación entre la AF y la salud mental; ya que esta mejora la circulación sanguínea cerebral y a través de influencia en estructuras centrales genera un impacto positivo en el control fisiológico del estrés(75).

La alimentación saludable, es importante en cualquier etapa de la vida y particularmente en condiciones en las que la demanda calórica puede ser menor. Sin embargo, en países como Colombia, en los que según el Departamento Administrativo Nacional de Estadística, para el 2018 existían regiones hasta con $95,5 \%$ de su población en condición de pobreza(76), y ante la crisis económica de muchas familias dada por la pandemia, puede ser difícil acceder a una alimentación variada y saludable, razón por la cual se recomienda que los productos que consumir se adecuen a las posibilidades de cada persona, sin dejar de considerar que alimentos como las frutas y las verduras, deberán preferirse, sobre los enlatados, los embutidos y las bebidas gaseosas que no tienen aporte nutricional(46).

Los altos volúmenes de información que circulan diariamente a través de medios físicos y virtuales, sobrepasan la capacidad individual de revisión y análisis, y señalan la necesidad de contar con alternativas como las revisiones de tema, revisiones de literatura, revisiones narrativas, revisiones sistemáticas, que con diferentes niveles de alcance procuran sintetizar información, lo cual agiliza los procesos para adquirir información relevante(77). Esta revisión presenta algunas de las recomendaciones a tener en cuenta para evitar el desacondicionamiento físico, y es posible que otras de interés para la población general, necesiten ser estudiadas en mayor detalle con fuentes de información complementarias.

Sin embargo, las recomendaciones incluidas, han sido revisadas por un grupo de expertos en el área de la fisioterapia, del movimiento corporal humano y en las estrategias para mantenerlo como el elemento esencial de salud que es; por tanto, se ha incorporado información actualizada, proveniente de la evidencia científica y de calidad. A pesar de esto, su alcance es descriptivo y en caso de requerir consideraciones para poblaciones particulares o casos específicos, será importante consultar con personal o entidades expertas en cada temática.

Se recomienda continuar con procesos que propendan por consolidar información para la población general, que le sirva de guía a las personas en el desarrollo de sus actividades durante y después de la pandemia y que procuren disminuir los efectos negativos asociados a las estrategias implementadas para disminuir el contagio.

\section{Conclusiones}

Esta revisión permitió presentar recomendaciones prácticas y de bajo costo, fáciles de aplicar en casa, basadas en la evidencia, con el fin de mitigar el impacto negativo en términos de desacondicionamiento e inactividad física, conductas sedentarias, malnutrición y estrés, causados por el aislamiento social obligatorio, como medida de contención no farmacológica para evitar el contagio por coronavirus.

Para evitar el desacondicionamiento físico, asociado al aislamiento social, es importante incorporar estrategias relacionadas con la AF, así como algunas recomendaciones nutricionales y de descanso adecuado. Incorporar estrategias que disminuyan el estrés y la ansiedad también contribuirán en el mantenimiento de la condición física y conservarlas en el tiempo, implica que se involucren dentro de un plan estructurado que permita el desarrollo de diferentes actividades, dentro de horarios determinados y acorde a la capacidad de cada individuo. Esto, puede traer beneficios inmediatos en la reducción del riesgo de infección y mejorar la calidad de vida en familia, y otros en el mediano y largo plazo, como constituir un estilo de vida saludable como elemento protector de enfermedades crónicas e infecciosas.

\section{Referencias}

1. Organización Mundial de la Salud (OMS). Alocución de apertura del Director General de la OMS en la rueda de prensa sobre la COVID-19 celebrada el 11 de marzo de 2020 [Internet]. Disponible en: https://www.who.int/es/dg/speeches/detail/whodirector-general-s-opening-remarks-at-the-mediabriefing-on-covid-19---11-march-2020

2. Universidad de Antioquia. Síntesis rápida: Intervenciones no farmacológicas para la contención, mitigación y 
supresión de la infección por COVID-19. Respuesta de 10 días. [Internet]. 2020. Disponible en: https://www.researchgate.net/publication/340265751_I ntervenciones_no_farmacologicas_para_la_contencion_mit igacion_y_supresion_de_la_infeccion_por_COVID-19

3. Ministerio de Salud y Protección Social de Colombia 2020. Resolución número 385 del 12 de marzo de 2020. Ministerio de Salud y Protección Social de Colombia. Bogotá, Colombia. [Internet]. Disponible en: https://www.minsalud.gov.co/sites/rid/Lists/Biblioteca Digital/RIDE/DE/DIJ/resolucion-385-de-2020.pdf

4. Centers for Disease Control and Prevention. Social Distancing, Quarantine, and Isolation. [Internet]. Atlanta (EU): CDC; 2020 Feb. Disponible en: https://www.cdc.gov/coronavirus/2019-ncov/preventgetting-sick/social-distancing.html

5. Chen P, Mao L, Nassis GP, Harmer P, Ainsworth BE, Li F. Coronavirus disease (COVID-19): The need to maintain regular physical activity while taking precautions. J Sport Health Sci [Internet]. 2020;9(2):103-4. Disponible en: https://www.ncbi.nlm.nih.gov/pmc/articles/PMC703177 $1 /$

6. Lee I-M, Shiroma EJ, Lobelo F, Puska P, Blair SN, Katzmarzyk PT, et al. Effect of physical inactivity on major non-communicable diseases worldwide: an analysis of burden of disease and life expectancy. Lancet [Internet]. 2012;380(9838):219-29. Disponible en: https://www.thelancet.com/journals/lancet/article/PIIS 0140-6736(12)61031-9/fulltext

7. Gené-Badia J, Ruiz-Sánchez M, Obiols-Masó N, Oliveras Puig L, Lagarda Jiménez E. Aislamiento social y soledad: ¿qué podemos hacer los equipos de atención primaria? Aten Primaria [Internet]. 2016;48(9):604-9. Disponible en:

https://www.ncbi.nlm.nih.gov/pmc/articles/PMC687784 0/

8. Enciso-Olivera CO, Galvis-Rincón JC, Díaz EDLT, DeviaLeón A, Camargo-Puerto DA. Efectos cardiovasculares de un protocolo de reacondicionamiento físico en pacientes críticos de tres centros asistenciales en Bogotá, Colombia. Medicas UIS [Internet]. 2016;29(2):161-73. Disponible en: http://www.scielo.org.co/scielo.php?script=sci_abstract\& pid $=$ S0121-

03192016000200015\&lng=en\&nrm=iso\&tlng=es

9. Maher JL, McMillan DW, Nash MS. Exercise and HealthRelated Risks of Physical Deconditioning After Spinal Cord Injury. Top Spinal Cord Inj Rehabil [Internet]. 2017;23(3):175-87. Disponible en: https://www.ncbi.nlm.nih.gov/pubmed/29339894

10. Sallis J. Physical activity, inmmunity, Inflammation and COVID-19 [Internet]. Disponible en: https://www.youtube.com/watch?v=F4mcbi9tD-

M\&feature=youtu.be

11. Zhang Y, Ma ZF. Impact of the COVID-19 Pandemic on Mental Health and Quality of Life among Local Residents in Liaoning Province, China: A Cross-Sectional Study. International Journal of Environmental Research and Public Health [Internet]. 2020;17(7):2381. Disponible en: https://www.mdpi.com/1660-4601/17/7/2381

12. American Collegue of Sports Medicine. ACSM's Guidelines for Exercise Testing and Prescription 10th ed. [Internet]. Indianapolis (Estados Unidos): ACSM; 2018. Disponible en: http://www.acsm.org/read-research/books/acsmsguidelines-for-exercise-testing-and-prescription
13. Morawska L, Cao J. Airborne transmission of SARS-CoV-2: The world should face the reality. Environ Int [Internet]. 2020;139:105730. Disponible en: https://www.sciencedirect.com/science/article/pii/S016 041202031254X

14. Leiva AM, Martínez MA, Cristi-Montero C, Salas C, Ramírez-Campillo $R$, Díaz Martínez $X$, et al. El sedentarismo se asocia a un incremento de factores de riesgo cardiovascular y metabólicos independiente de los niveles de actividad física. Revista médica de Chile [Internet]. 2017;145(4):458-67. Disponible en: https://scielo.conicyt.cl/scielo.php?script=sci_abstract\&pi $\mathrm{d}=\mathrm{S} 0034$ -

98872017000400006\&lng=es\&nrm=iso\&tlng=es

15. Owen N, Healy GN, Matthews CE, Dunstan DW. Too much sitting: the population health science of sedentary behavior. Exercise and sport sciences reviews. 2010;38(3):105-13. Disponible en: https://www.ncbi.nlm.nih.gov/pmc/articles/PMC340481 5/.

16. Arocha Rodulfo JI. Sedentarismo, la enfermedad del siglo XXI. Clínica e Investigación en Arteriosclerosis [Internet]. 2019;31(5):233-40. Disponible en: http://www.sciencedirect.com/science/article/pii/S0214 916819300543

17. World Health Organization (WHO). Stay physically active during self-quarantine [Internet]. Dinamarca: WHO; 2019. Disponible en: https://www.euro.who.int/en/healthtopics/health-emergencies/coronavirus-covid-

19/technical-guidance/stay-physically-active-during-selfquarantine

18. Lisbona Catalán A, Palma Milla S, Parra Ramírez P, Gómez Candela C. Obesidad y azúcar: aliados o enemigos. Nutrición Hospitalaria [Internet]. 2013;28:81-7. Disponible http://scielo.isciii.es/scielo.php?script=sci_abstract\&pid= S0212-16112013001000010\&lng=es\&nrm=iso\&tlng=es

19. Organización Mundial de la Salud. Obesidad y sobrepeso [Internet]. Ginebra (Suiza): OMS; 2020. Disponible en: https://www.who.int/es/news-room/factsheets/detail/obesity-and-overweight

20. Simpson R, Campbell John P, Gleeson M, Krüger K, Nieman $D$, Pyne D, et, al. Can Exercise Affect Immune Function to Increase Susceptibility to Infection? Exercise Immunology Review [Internet]. 2020;26:8-22. Disponible en: https://www.ncbi.nlm.nih.gov/pubmed/32139352

21. Organización Mundial de la Salud. La obesidad entre los niños y los adolescentes se ha multiplicado por 10 en los cuatro últimos decenios [Internet]. Ginebra (Suiza): OMS; 2020. Disponible en: https://www.who.int/es/newsroom/detail/11-10-2017-tenfold-increase-in-childhoodand-adolescent-obesity-in-four-decades-new-study-byimperial-college-london-and-who

22. Vallejo- Figueroa PE, Sánchez-Barrera IC, ArciniegasBarrera J, Escobar-Díaz F., editores. Obesidad infantil: una amenaza silenciosa [Internet]. Ministerio de Salud y Protección Social; 2019 [citado 2020 Mar 9]. Disponible en:

https://www.minsalud.gov.co/sites/rid/Lists/Biblioteca Digital/RIDE/DE/PES/resumen-politica-obesidadinfantil-amenaza-silenciosa.pdf

23. Altena E, Baglioni C, Espie CA, Ellis J, Gavriloff D, Holzinger $B$, et al. Dealing with sleep problems during home confinement due to the COVID-19 outbreak: practical 
recommendations from a task force of the European CBT-I Academy. J Sleep Res [Internet]. 2020; 4e 13052. Disponible

en: https://www.ncbi.nlm.nih.gov/pubmed/32246787

24. Asensi L., editor Coronavirus: Efectos psicológicos generados por el confinamiento. [Internet]. Madrid: Centro Psicológico Madrid; 2020 [citado 2020 Mar 10]. Disponible

en: https://www.psicologiamadrid.es/blog/articulos/psicolo gia-y-coronavirus/coronavirus-efectos-psicologicosgenerados-por-el-confinamiento

25. Wang C, Pan R, Wan X, Tan Y, Xu L, Ho CS, et al. Immediate Psychological Responses and Associated Factors during the Initial Stage of the 2019 Coronavirus Disease (COVID19) Epidemic among the General Population in China. Int J Environ Res Public Health [Internet]. 2020;17(5). Disponible en: https://www.ncbi.nlm.nih.gov/pmc/articles/PMC708495 2/

26. Ministerio de Tecnologías de la Información y las Comunicaciones de Colombia. Aprenda a detectar y combatir las noticias falsas en internet [Internet]. Bogotá (Colombia): Minitic; 2019. Disponible en: https://www.mintic.gov.co/portal/inicio/Sala-dePrensa/Noticias/101931:Aprenda-a-detectar-y-combatirlas-noticias-falsas-en-internet

27. Nieman DC, Wentz LM. The compelling link between physical activity and the body's defense system. Journal of Sport and Health Science [Internet]. 2019;8(3):201-17. Disponible en: http://www.sciencedirect.com/science/article/pii/S2095 254618301005

28. Jones AW, Davison G. Chapter 15 - Exercise, Immunity, and Illness. En: Zoladz JA, editor. Muscle and Exercise Physiology [Internet]. Academic Press; 2019. p. 317-44. Disponible en: http://www.sciencedirect.com/science/article/pii/B978 0128145937000153

29. Organización Mundial de la Salud. Estrategia mundial sobre régimen alimentario, actividad física y salud [Internet]. Ginebra (Suiza): OMS; 2010. Disponible en: https://apps.who.int/iris/bitstream/handle/10665/4444 1/9789243599977_spa.pdf

30. Edwards JP, Walsh NP, Diment PC, Roberts R. Anxiety and perceived psychological stress play an important role in the immune response after exercise. Exerc Immunol Rev [Internet]. 2018;24:26-34. Disponible en: https://www.ncbi.nlm.nih.gov/pubmed/29461966

31. Walsh NP. Recommendations to maintain immune health in athletes. Eur J Sport Sci. [Internet]. 2018 Jul;18(6) [citado 2020 Feb 12]:820-831. doi: 10.1080/17461391.2018.1449895

32. King AC, Whitt-Glover MC, Marquez DX, Buman MP, Napolitano MA, Jakicic J, et al. Physical Activity Promotion: Highlights from the 2018 Physical Activity Guidelines Advisory Committee Systematic Review. Med Sci Sports Exerc [Internet]. 2019;51(6):1340-53. Disponible en: https://www.ncbi.nlm.nih.gov/pubmed/31095090

33. Hojman P. Exercise protects from cancer through regulation of immune function and inflammation. Biochem Soc Trans [Internet]. 2017;45(4):905-11. Disponible

https://www.ncbi.nlm.nih.gov/pubmed/28673937
34. Basso JC, Suzuki WA. The Effects of Acute Exercise on Mood, Cognition, Neurophysiology, and Neurochemical Pathways: A Review. Brain Plast [Internet]. 2(2):127-52. Disponible en: https://www.ncbi.nlm.nih.gov/pmc/articles/PMC592853 4/

35. Adam EK, Quinn ME, Tavernier R, McQuillan MT, Dahlke KA, Gilbert KE. Diurnal cortisol slopes and mental and physical health outcomes: A systematic review and metaanalysis. Psychoneuroendocrinology [Internet]. 2017;83:25-41. Disponible en: https://www.ncbi.nlm.nih.gov/pubmed/28578301

36. Department of Health and Human Services. Physical activity guidelines advisory Committee Scientific Report [Internet]. U.S.: ODPHP; Feb. Disponible en: https://health.gov/paguidelines/second-edition/report/.

37. BBC News. Coronavirus: 5 actividades que puedes hacer en casa para mantenerte en forma durante el confinamiento por el covid-19 [Internet]. BBC News. 2020 Abr 13; Mundo. Disponible en: https://www.bbc.com/mundo/noticias-52042625

38. Organización Mundial de la Salud. Prepare su lugar de trabajo para la COVID-19 [Internet]. Ginebra (Suiza): OMS; 2020 Feb. versión 1.4. Disponible en: https://www.paho.org/es/documentos/prepare-sulugar-trabajo-para-covid-19

39. Guillén M. Ergonomía y la relación con los factores de riesgo en salud ocupacional. Rev cubana enferm. 2006;22(4). Disponible en: http://scielo.sld.cu/scielo.php?script=sci_arttext\&pid=S0 864-03192006000400008\&lng=es

40. Matsudo SMM. Actividad Física: Pasaporte Para La Salud. Revista Médica Clínica Las Condes [Internet]. 2012;23(3):209-17. Disponible en: http://www.sciencedirect.com/science/article/pii/S0716 864012703036

41. Urdampilleta A, Martínez-Sanz JM, Julia-Sanchez S, Álvarez-Herms J. Protocolo de hidratación antes, durante y después de la actividad físico-deportiva. Motricidad European Journal of Human Movement [Internet]. 2013;31:57-76. Disponible en: https://www.redalyc.org/articulo.oa?id=274229586004

42. Hospital Vall d' Hebron. Coronavirus SARS-CoV-2: ¿Cómo mantenerse en forma durante el confinamiento? [Internet]. Barcelona (España): Hospital Vall d' Hebron; 2018. Disponible en: https://hospital.vallhebron.com/es/consejos-desalud/coronavirus-sars-cov-2-como-mantenerse-enforma-durante-el-confinamiento

43. De Souto P. Time to Challenge Public Health Guidelines on Physical Activity. Sports Medicine. 2015;45(6):769-73. Disponible

https://www.ncbi.nlm.nih.gov/pubmed/25833079

44. Barreto P de S. Why are we failing to promote physical activity globally? Bulletin of the World Health Organization. 1 de junio de 2013;91(6):390-390A. [Internet]. Disponible en: https://www.ncbi.nlm.nih.gov/pubmed/24052671

45. Xu K, Cai H, Shen Y, Ni Q, Chen Y, Hu S, et al. [Management of corona virus disease-19 (COVID-19): the Zhejiang experience]. Zhejiang Da Xue Xue Bao Yi Xue Ban [Internet]. 2020;49 [citado 2020 Mar];(1). Disponible en: https://www.ncbi.nlm.nih.gov/pubmed/32096367 
46. Baladia E, Martínez Rodríguez R. Legumbres y salud: sumario de evidencias rápidas. RED -NuBE; 2016. Disponible

en: http://www.ub.edu/campusalimentacio/ca/documents/I nnovant\%20llegums/legumbres_2018.pdf.

47. World Health Organization. Healthy diet [Internet] Ginebra (Suiza): WHO; 2018. Disponible en: https://www.who.int/news-room/factsheets/detail/healthy-diet

48. Organización Mundial de la Salud (OMS). Biblioteca electrónica de documentación científica sobre medidas nutricionales (eLENA) [Internet]. 2019. Disponible en: https://www.who.int/elena/titles/sodium_cvd_adults/es /

49. Organización Mundial de la Salud. Nutrición: Ingesta de azúcares para adultos y niños [Internet]. Ginebra (Suiza). OMS; 2015. Disponible en: https://www.who.int/nutrition/publications/guidelines/ sugars_intake/es/

50. Reis AMD, Fructhenicht AVG, Moreira LF. NUTRIC score use around the world: a systematic review. Rev Bras Ter Intensiva [Internet]. 2019;31(3):379-85. Disponible en: https://www.ncbi.nlm.nih.gov/pubmed/31618358

51. Congote Hurtado S. Sistema de cocción al vapor con piedras volcánicas. 2011; Disponible en: http://repositorio.ucp.edu.co/handle/10785/739

52. Academia Española de Nutrición y Dietética. Consejo General de Colegios Oficiales, de Dietistas-Nutricionistas. Recomendaciones de alimentación y nutrición para la población española ante la crisis sanitaria del COVID-19. $2020 . \quad$ Disponible en: https://academianutricionydietetica.org/NOTICIAS/alim entacioncoronavirus.pdf.

53. National Institute of Mental health. 5 cosas que usted debe saber sobre el estrés [Internet]. Disponible en: https://www.nimh.nih.gov/health/publications/espanol/ 5-cosas-que-usted-debe-saber-sobre-elestres/index.shtml\#pub4

54. Herguedas AJU. La meditación como práctica preventiva y curativa en el sistema nacional de salud. Medicina naturista [Internet]. 2018;12(1):47-53. Disponible en: https://dialnet.unirioja.es/servlet/articulo?codigo $=6267$ 068

55. Organización Mundial de la Salud. Brote de enfermedad por coronavirus (COVID-19): orientaciones para el público. [Internet]. Ginebra (Suiza). OMS; 2020 Disponible en:

https://www.who.int/es/emergencies/diseases/novelcoronavirus-2019/advice-for-public

56. Borquez P. Calidad de sueño, somnolencia diurna y salud autopercibida en estudiantes universitarios. Eureka (Asunción) en Línea [Internet]. 2011;8(1):80-90. Disponible en: http://pepsic.bvsalud.org/scielo.php?script=sci_abstract \&pid=S2220

$90262011000100009 \& \operatorname{lng}=$ pt\&nrm=iso\&tlng=es

57. González LE. Cambios del modo y estilo de vida; su influencia en el proceso salud-enfermedad. Rev Cuba Estomatol. 6 de septiembre de 2004;41(3):54-61. Disponible en: http://scielo.sld.cu/scielo.php?script=sci_arttext\&pid=S0 034-75072004000300009

58. Catalán T, Gallego-Gómez J, González-Moro M, SimonelliMuñoz A. Factores que influyen en la calidad del sueño de pacientes institucionalizados. Revista Portuguesa de Enfermagem de Saúde Mental [Internet]. 2014 [cited 2020 Mar];12:[43-50]. Disponible en: http://www.scielo.mec.pt/scielo.php?script=sci_abstract \&pid=S1647-21602014000300006\&lng=es\&nrm=iso

59. Roy D, Tripathy S, Kar SK, Sharma N, Verma SK, Kaushal V. Study of knowledge, attitude, anxiety \& perceived mental healthcare need in Indian population during COVID-19 pandemic. Asian J Psychiatr [Internet]. 2020;51:102083. Disponible

https://www.ncbi.nlm.nih.gov/pubmed/32283510

60. Murillo R, Pirzada A, Wu D, Gallo LC, Davis S, Ostrovsky NW, et al. The Association Between Family Social Network Size and Healthy Lifestyle Factors: Results from the Hispanic Community Health Study/Study of Latinos (HCHS/SOL). J Behav Med [Internet]. 2020;43(2):198208. Disponible en: http://link.springer.com/10.1007/s10865-019-00082-9

61. Okezue OC, Nwafor GC, Ezeukwu OA, John JN, Uchenwoke CI. Adherence to Home Exercise Programmes and its Associated Factors among Patients Receiving Physiotherapy. clinhp [Internet]. 2019;9(1):7-14. Disponible en: https://docs.wixstatic.com/ugd/7b6489_876d4a2ced684 618af5c14fdda65eff7.pdf

62. Periódico la República. El teletrabajo hace que las personas le dediquen más horas a la jornada laboral [Internet]. Periódico la República. 2020 Disponible en: https://www.larepublica.co/empresas/el-teletrabajohace-que-las-personas-le-dediquen-mas-horas-a-lajornada-laboral-2983857

63. Segura H, Hernández D. Teletrabajo, prioridad más allá de la pandemia. UdeA Noticias 2020 Abr 7; Sección Sociedad. Disponible en: http://www.udea.edu.co/wps/portal/udea/web/inicio/u dea-noticias/udeanoticia/!ut/p/z0/fUw9DwExGP4rlhsvLc5hvBgkYjCI3HW Rx7Xhpe1bvRI_32EQi-

XJ8y2UqIXyuNMRidjD9rpR5X42X4yGVSHXsixKWZWbYjI dLcfbnRQrof4X-gc6X6qEqpln8wjiTpwTLA3bZBJdL_qxM58-

AsHnh01hC6T77Unza_W1w4mEmtqGdbBIZmYyWSsSRE HnDkPfRxJQ-cOXQ5rkQd4bRxBhItqnlPk07Y!/

64. Brooks SK, Webster RK, Smith LE, Woodland L, Wessely S, Greenberg N, et al. The psychological impact of quarantine and how to reduce it: rapid review of the evidence. The Lancet [Internet]. 2020;395(10227):912-20. Disponible en:

https://linkinghub.elsevier.com/retrieve/pii/S01406736 20304608

65. University of Rochester Medical Center. Preventing Sports Injuries [Internet]. Disponible en: urmc.rochester.edu/encyclopedia/content.aspx?contentty peid $=85 \&$ contentid $=$ P00935

66. Duperly J, Lobelo F. Prescripción del ejercicio: una guía para recomendar actividad física a cada paciente [Internet]. Bogotá, D.C.: Ediciones de la U; 2015. Disponible en: https://edicionesdelau.com/producto/prescripcion-delejercicio-una-guia-para-recomendar-actividad-fisica-acada-paciente/

67. Simpson RJ. American Collegue of sports medicine ACSM's. Ejercicio, inmunidad y la pandemia del COVID-19 [Internet]. 2020. Disponible en: 
https://www.acsm.org/blog-detail/acsm-

blog/2020/04/16/ejercicio-inmunidad-y-la-pandemia-

del-covid-19

68. Pearson N, Braithwaite RE, Biddle SJH, van Sluijs EMF, Atkin AJ. Associations between sedentary behaviour and physical activity in children and adolescents: a metaanalysis. Obes Rev [Internet]. 2014;15(8):666-75. Disponible en:

https://www.ncbi.nlm.nih.gov/pubmed/24844784

69. Domingues-Montanari S. Clinical and psychological effects of excessive screen time on children. J Paediatr Child Health [Internet]. 2017;53(4):333-8. Disponible en: https://www.ncbi.nlm.nih.gov/pubmed/28168778

70. Blog British Journal of Sports Medicine [Internet].Gran Bretaña 2020 [cited 2020 Mar 13]. Disponible en: https://blogs.bmj.com/bjsm/2020/04/08/for-the-atrisk-or-vulnerable-covid-19-group-staying-or-becomingactive-during-social-distancing/

71. Moncada LVV, Mire LG. Preventing Falls in Older Persons. Am Fam Physician [Internet]. 2017;96(4):240-7. Disponible en: https://www.ncbi.nlm.nih.gov/pubmed/28925664

72. Ministerio de Salud y Protección Social de Colombia. Orientaciones para la prevención, contención y mitigación del coronavirus COVID-19 en personas adultas mayores, centros vida, centros día y centros de protección de larga estancia para adultos mayores [Internet]. Bogotá (Colombia): Ministerio de Salud; 2020 Mar. ASIF13Versión $1 . \quad$ Disponible en: https://www.minsalud.gov.co/sites/rid/Lists/Biblioteca Digital/RIDE/DE/PS/asif13-adulto-mayor.covid-19.pdf

73. Taylor S. The Psychology of Pandemics Preparing for the Next Global Outbreak of Infectious Disease [Internet]. Cambridge Scholars Publishing; 2019. 178 p. Disponible en: https://www.cambridgescholars.com/thepsychology-of-pandemics

74. Mikkelsen K, Stojanovska L, Polenakovic M, Bosevski M, Apostolopoulos V. Exercise and mental health. Maturitas [Internet]. 2017;106:48-56. Disponible en: https://www.ncbi.nlm.nih.gov/pubmed/29150166

75. Pérez Tapia, C. La actividad física como terapia complementaria en las enfermedades de salud mental. Rev. peru. cienc. act. fís. deporte [Internet]. 2020 Ene [Citado 2020 Feb]; 7(1): 879-890. Disponible en: https://www.rpcafd.com/index.php/rpcafd/article/view $/ 79 / 110$

76. Departamento Administrativo Nacional de Estadística. Medida de pobreza multidimensional municipal de fuente censal 2018 [Internet]. Bogotá (Colombia); DANE; 2020 Feb. Disponible en: https://www.dane.gov.co/index.php/estadisticas-portema/pobreza-y-condiciones-de-vida/pobreza-ydesigualdad/medida-de-pobreza-multidimensional-defuente-censal

77. Araujo M. Fundamentos y limitaciones de la revisión de la literatura biomédica. Medwave [Internet]. 2011;11(10). Disponible en: https://www.medwave.cl/link.cgi/Medwave/Series/mbe $01 / 5194$ 